\title{
An Overview of Performance Enhancing Drugs (PED's) in Sports and WADA
}

\author{
Karri Sowjanya, Chandrashekaran Girish* \\ Department of Pharmacology, Jawaharlal Institute of Postgraduate Medical Education and Research (JIPMER), Puducherry, INDIA.
}

\begin{abstract}
The use of certain banned and illegal substances either to improve one's cognitive skills or to enhance their performance by athletes has become widely prevalent. These substances are commonly referred as performance enhancing drugs or PED's and are used without any therapeutic intent. They belong to a wide variety of classes, some of which include the anabolic steroids, hormonal agents, diuretics and the CNS stimulants. The WADA (World Anti-Doping Agency), a collaborated network between the sports authorities and the global governments was established to fight against doping so as to have a fair competition among the athletes. Although, the regulatory bodies have put forth stringent legislations that can prevent their misuse and safeguards the athletes against the adverse effects of such drugs, it has become rather difficult to keep doping under check as the recent developments have resulted in the emergence of so called designer drugs that are difficult to detect with the available analyti-
\end{abstract}

cal techniques. Subsequently, WADA came up with the concept of ABP (Athlete Biological Passport) which helped resolve some of these issues. This review tries to look into PED's, the way they act, their ill-effects and also in brief about the designer drugs. It also highlights the role of WADA, $\mathrm{ABP}$ and the prohibited list of substances and methods for the year 2019. Key words: Anabolic steroids, Erythropoietin, World Anti-Doping Agency, Athlete Biological Passport, CNS stimulants.

\section{Correspondence}

Dr. Chandrashekaran Girish, Department of Pharmacology, Jawaharlal Institute of Postgraduate Medical Education and Research (JIPMER), Puducherry, INDIA.

Phone: +91 04132272380

Email: gcnx@rediffmail.com

DOI: 10.5530/jyp.2019.11.71

\section{INTRODUCTION}

Performance Enhancing Drugs (PED's) refers to drugs that have a potential to alter the human body and its biological functions so as to improve his/her own performance. ${ }^{1}$ These drugs do not alone restrict to the improvement of muscle mass or muscle strength; but also serve as a cognitive enhancer in terms of memory, intelligence and creativity. This has resulted in their widespread use for one's self-improvement in nonmedical indications. In the recent years, the usage of PED's has tremendously increased not only among athletes, but also among the teenagers and young adults to improve their overall performance. Though they are quite beneficial in several regards, the mishaps that can occur in the long run and also their unfair advantage when used in athletes should be taken into consideration. PED's, when used in athletes are commonly referred to as ergogenic aids. ${ }^{2}$

\section{COMMONLY USED DRUG CLASSES OF PED'S}

The performance enhancing drugs belong to a wide variety of classes like the anabolic steroids, hormonal agents, diuretics, CNS (Central nervous system) stimulants and many others which are enlisted in Table $1 .^{3-6}$

\section{Anabolic agents}

The anabolic agents include the AAS with combined anabolic and androgenic action; and others like clenbuterol with anabolic and $\beta_{2}$ agonistic action. The anabolic action of AAS promotes muscle building while the androgenic action is responsible for the development of masculine characters like facial hair and deepening of voice. ${ }^{4}$ The mechanism of action of AAS is shown in Figure 1. On the other hand, clenbuterol is found to increase the muscle strength when given systemically. However, its overall action on whether it can improve the performance in athletes is not yet clear. The sports agencies have presently put a ban on the usage of $\beta_{2}$ agonists systemically till proven to be fair enough.?

Detection: Testosterone undecanoate is the most common form abused by athletes. It can be assessed by measuring the changes of plasma and urine steroid levels. After its oral or intramuscular administration, the glucuronidated metabolites like androsterone, 5-dihydrotestosterone and etiocholanolone are elevated in plasma. The ratios of Testosterone Glucuronide(TG)/unconjugated Testosterone(T), TG/17Hydroxyprogesterone(17OHP) and TG/Luteinizing Hormone(LH) in plasma are considered to be the most sensitive markers to detect exogenous testosterone administration. Also, the ratio of urine testosterone to $\mathrm{LH}$ more than 30 is considered to be a positive finding in the doping test of athletes as exogenous testosterone suppresses LH. Another way is to calculate the ratio of TG to Epitestosterone Glucuronide (EG). A cutoff value of 6 is considered to be positive as the usage of anabolic steroids suppresses the endogenous testosterone secretion. ${ }^{8,9}$

As testosterone is an endogenous compound, illicit use of such compounds is rather a tough job to prove. So, the isotope ratio mass spectrometry techniques $\left({ }^{12} \mathrm{C}:{ }^{13} \mathrm{C}\right)$ were developed to distinguish between the exogenous and endogenous steroids. And also, as they are used routinely during the entire training period, an out-of-competition testing is quite beneficial rather than its testing only during the event. ${ }^{3,9}$

Adverse effects: The most common adverse effects include abnormalities in lipid profile (decreased high density lipoproteins and increased low density lipoprotein) and hepatic enzymes (increased aspartate aminotransferase); and mood disturbances (irritability, psychotic symptoms and depression). ${ }^{10}$

The serious adverse effects include infertility in males, masculinisation in females, cardiovascular risks, tumours of liver and kidney and 
premature skeletal maturation. ${ }^{3}$ Other less common ones include acne, alopecia, injection site reactions, increased haematocrit and decreased testicular size. ${ }^{10}$

\section{Hormonal substances}

The most common hormones and other associated substances that can be used as PED's include erythropoietin, growth hormone, insulin and Human Chorionic Gonadotropin (hCG).

\section{Erythropoietin (EPO)}

It is a glycoprotein produced by kidney (juxtaglomerular cells) and macrophages. It can be given subcutaneously, intravenously or intraperitoneally and is indicated to treat anaemia in conditions of chronic renal failure, in cancer chemotherapy and in AIDS patients. ${ }^{11}$ EPO causes the stimulation of erythroid precursors to generate red blood cells that increase the delivery of oxygen to tissues. So, the Recombinant $\mathrm{Hu}$ man EPO (rHuEPO) was developed and used widely among athletes as a means of blood doping in endurance sports resulting in its ban in 1990. ${ }^{12}$ Detection: rHuEPO misuse in sports was detected either by indirect methods (secondary blood markers) or direct methods (rHuEPO in urine). Multiple blood markers were considered like the haematocrit level, haemoglobin level, reticulocyte count, EPO concentrations and the percentage of macrocytic cells. The rHuEPO in urine was detected by isoelectric focusing method based on the concept that it is less acidic when compared to endogenous EPO. However, due to its short half-life, it was later found that it is difficult to detect when the drug usage has ceased for more than 7 days. ${ }^{12}$ Adverse effects: There is an increased viscosity of blood resulting in hypertension, thrombosis and other neurological diseases. ${ }^{3}$

\section{Human growth hormone $(\mathrm{HGH})$}

It is an anabolic hormone that increases the nitrogen levels thereby promoting protein synthesis leading to muscle growth. It also acts on the adipose tissue causing lipolysis; and diverting the energy that is liberated for the synthesis of proteins and carbohydrates. Apart from these actions, it causes a release of IGF-I (insulin like growth factor-1) and procollagen type III amino-terminal propeptide (P-III-NP) that further enhances the muscle growth and strength. This data from various in vitro and in vivo studies has gained popularity to use recombinant HGH by athletes for muscle building and to improve their physical performance. However, the benefit of using it as an ergogenic aid is still controversial. ${ }^{13}$ Detection: The direct method to evaluate doping is by the GH isoform test. This was framed to differentiate between the exogenous and endogenous GH in blood based on the available isoforms (recombinant HGH exist in a single isoform whereas the endogenous form exists in 3 different isoforms that vary in their molecular weight). The drawback associated with this method is the difficulty in its detection after $24 \mathrm{hr}$ of last dose due to its short half-life.

This has led to the development of specific biomarker tests to measure the levels of IGF-I and P-III-NP in blood by immunoassay. The levels of IGF-I can be detected till one week and those of P-III-NP for about 4-6 weeks. So, there is a wide opportunity for their detection in the doping tests but are comparatively less specific with limitations in the available assays and also due to the associated variations with respect to age and sex among different individuals. Also the out-of-competition testing needs to be done. The novel genomic and proteomic techniques are being tried to develop a signature that can indicate exogenous $\mathrm{GH}$ use so as to build a robust test in doping. However, their detection in other bodily fluids like urine and saliva were not successful. ${ }^{3,13,14}$

Adverse effects: The long term adverse effects include acromegalic changes (jaw overgrowth, frontal bossing, dental malocclusion, spinal stenosis etc), arthralgias, myalgias, insulin resistance leading to diabetes, cardiovascular problems (hypertension, dilated cardiomyopathy), cancer risk (colorectal, prostate, breast, thyroid cancers). The incidence of these adverse effects varies with the age of the individual, the dose taken and the duration ${ }^{13,14}$

\section{Insulin}

It is an anabolic hormone that promotes muscle growth by increasing the levels of nitrogen in muscle similar to growth hormone. When short acting insulin is administered along with glucose, it was found to increase the glucose uptake thereby helping in energy production. It is mostly used by body builders and weight lifters. However, its effect on improving the overall performance in athletes is not well established. It can be detected in doping tests by measuring the insulin analogs in urine through mass spectroscopy for the observation of a characteristic spectrum that is distinguishable from the human insulin. The most common side effects documented include hypoglycaemia and weight gain.,14

\section{Human chorionic gonadotropin (hCG) and luteinizing hormone (LH)}

They are heterodimeric proteins composed of $\alpha$ and $\beta$ subunits and exist in various molecular forms like intact and nicked forms, dissociated forms and degradation products. hCG and LH act by binding to Chorioganadotropin (CG) and $\mathrm{LH}$ receptor respectively and mediate the release of testosterone from Leydig cells which can help in muscle building and performance. Their usage is banned in male athletes alone as testosterone production in females was found to be negligible. Also, the usage of other substances like anti-estrogens and aromatase inhibitors that can release endogenous LH are also prohibited. ${ }^{15}$ They are highly expensive and need the administration of several injections in a week. Despite all these concerns, their true effect on the improvement of muscle strength is still doubtful. ${ }^{9}$

Their illicit use can be detected from the urine samples. To detect hCG in urine, immunoassays are carried out initially as screening tests followed by their confirmation with mass spectrometry. If $\mathrm{hCG} \alpha / \beta$ heterodimer levels are $>5$ IU/L by immunoassays or $>2$ IU/L by mass spectroscopic assays, it is said to be positive. For LH, immunological assays are done and if it is $>60 \mathrm{IU} / \mathrm{L}$ by Immulite assay or $>4$ IU/L by Delfia assay, it is considered to be a positive finding in the doping tests. ${ }^{9,15}$

\section{CNS Stimulants}

This class of drugs can be used in dual ways either as a cognition enhancer (nootropics or smart drugs) or for performance enhancement in athletes. They are found to improve performance in non-endurance sports like swimming, sprinting and weight lifting. The most common drugs abused include caffeine, ephedrine, pseudoephedrine and amphetamine. They mainly act by releasing neurotransmitters like dopamine, serotonin and noradrenaline that increase the metabolism thereby reducing fatigue and causing aggressiveness and competitiveness. However, there are some studies stating that the effect is mainly psychological. ${ }^{3,16}$ The urine samples of the athletes are confirmed with doping by techniques like gas chromatography or Liquid Chromatography (LC) Mass Spectrometry (LC-MS/MS). The common adverse effects include hyperthermia, dehydration, hypertension, coronary insufficiency, cutaneous vasoconstriction and even death. ${ }^{3,16}$

\section{$\beta_{2}$ agonists}

Inhaled $\beta_{2}$ agonists were tried as an ergogenic aid in athletes like cyclists, runners, skiers and swimmers. In some studies, there is an improvement in lung function characterised by an increase in FEV1 with mild bronchodilator effect. However, it has not shown any improvement in their physical performance. However, they are still included in the list of prohibited substances with the exception of salbutamol, terbutaline, salmeterol and formeterol usage in asthmatic athletes. In these special 
situations, a TUE (Therapeutic Use Exemption) signed by the athlete and the physician needs to be submitted to the concerned anti-doping authorities along with the lung function reports. If the urinary concentration of salbutamol is $>1000 \mathrm{ng} / \mathrm{ml}$, it is said to be a positive doping test as it is highly difficult to attain such high concentrations through inhalation alone. ${ }^{17}$

\section{$\beta$ blockers}

The drugs belonging to this class like propranolol, atenolol, acebutolol and many others are prohibited for their usage in athletes in precision sports like archery, shooting, golf and billiards. It is found that it helps in reducing the tremor and anxiety of the athletes during in-competition that can help improve their performance. The levels of drug and/or its metabolites in the urine are measured to confirm doping. ${ }^{3}$

\section{Diuretics}

The drugs belonging to different classes like loop diuretics, thiazide diuretics, osmotic diuretics and mineralocorticoid receptor antagonists are being abused by athletes in various sports. They cause the loss of water from the body and are hence misused for rapid weight loss. This practice is commonly followed by boxers before weighing in. Also, it acts as a masking agent by preventing the detection of other illicit substances like anabolic steroids or CNS stimulants as the urine is diluted. So, the WADA has included diuretics in the prohibited list of substances during in-competition and also out-competition. However, their role as a performance enhancer is very minimal. Their levels can be detected in urine with the help of LC/MS techniques. ${ }^{3,18}$

\section{Narcotics}

The consumption of narcotics is common among athletes involved in violent sports as it helps to mask or relieve the pain associated with injuries. The levels of narcotics in doping tests can be detected from their urine samples. On consumption of codeine, the levels of morphine in the urine are elevated. So, the morphine to codeine ratio is taken as the most common marker to assess its misuse in athletes. If the ratio is $<1$, it is due to the intake of only codeine; and if it is $>1$, it is due to the intake of either morphine or heroin. However, a cut-off value of 1 is not always absolute as some of the athletes with ultra-rapid CYP2D6 metabolism already have levels of $>1$ even with the intake of codeine alone. So, the pharmacogenomics testing for CYP2D6 metabolism should be done to prevent other legal issues.,19

\section{Blood Doping}

The most common method employed in blood doping is by means of blood transfusion before the competition. Previously, allogenic blood transfusion was widely followed by many athletes. However, with the development of flow cytometry techniques, it was very easy to identify the athletes who have received even one unit of allogenic blood. ${ }^{20}$ Because of these concerns, athletes started using their own blood (autologous blood transfusion) in which about 1-2 litres of blood was withdrawn and stored at suitable conditions, to be infused before the competition. This is found to increase the level of haemoglobin and in turn enhancing the oxygen delivery to the muscles, thereby improving their aerobic capacity and performance. It is rather difficult to identify this illicit practice when compared to allogenic blood doping. But presently, the ratio of mass of Haemoglobin $(\mathrm{Hb})$ in the mature erythrocyte to that in the reticulocytes $\left(\mathrm{RBC}_{\mathrm{Hb}} / \mathrm{Ret}_{\mathrm{Hb}}\right.$ ratio) is considered to be one of the best indicators for detecting autologous blood doping. Another method for detection is by Carbon Monoxide ( $\mathrm{CO}$ ) rebreathing method in which the athlete is asked to inhale oxygen-carbon monoxide mixture to calculate the $\mathrm{Hb}$ mass. However, it is not acceptable as the inhalation of $\mathrm{CO}$ before the competition affects their performance. ${ }^{3,20}$
Secondly, the ESA's (erythropoiesis stimulating agents) like Erythropoietin (Epo), epoetin alfa, darbepoetin alfa and methoxy polyethylene glycol epoetin beta were abused by many athletes to enhance their performance. This can be detected by increased levels of immature reticulocytes due to their accelerated release from the bone marrow with an associated increase in the life span..$^{20}$ Also, the artificial oxygen carriers like perfluorocarbon emulsions (oxygen, oxyfluor) were used as a PED. On intravenous administration, the droplets are taken by the reticuloendothelial system and improved the tissue oxygenation, thereby enhancing the performance. The common side effects include flu like symptoms and febrile reactions; but with higher doses, transient thrombocytopenia is evident in a few cases. It is difficult to detect this substance in doping tests as it is mainly expelled out from the body by breathing; and there are no changes observed in the blood or urine tests. ${ }^{21}$

\section{Gene Doping}

The gene transfer techniques were initiated with the aim of preventing hereditary diseases and to cure diseases that lack proper treatment like HIV, cancer and haemophilia. While performing studies, some genes that can enhance the performance of individuals were found out due to their effects on body weight, size and aerobic capacity; and are referred to as sports genes. This was supported with the discovery of Mutations in Myostatin Gene (MSTN) and Erythropoietin (EPO) receptor gene (EPOR) that can affect the performance. EPOR increased the haemoglobin mass (>200 g/L) and haematocrit levels (to 68\%) enhancing the delivery of oxygen to muscles; implicating its advantage in elite sports. The other targets for gene doping include Vascular Endothelial Growth Factor (VEGF), insulin-like growth-factor-I (IGF-I) and follistatin (FST). The first thought of its misuse in sports arose when a German coach has tried to acquire a drug called Repoxygen containing EPO gene linked with the Adeno-Associated Virus (AAV) vector to improve the performance of his trainee. The authorities had then put a ban on the delivery of genes, genetic elements and nucleic acids for doping. The other vector systems commonly used are the Recombinant AAV (rAAV) and lentivirus systems; and do have a good efficacy and safety profile in trials. ${ }^{20,22}$

The newer approaches in the near future might target on the gene editing tools like CRISPR/Cas9 to modify the DNA in embryos or germ lines to enhance the performance. This can be done by introducing the desired genetic material into the embryos for designing babies with a future intent of bringing about high performance athletes. However, one of the major limitations is that, it cannot guarantee a high performance with the modification of only a fewer genes. Secondly, the off-target mutations and other side effects associated in the long run are often unpredictable. ${ }^{22}$

\section{Nutritional Supplements}

The nutritional supplements like creatine, ornithine alpha ketoglutarate, proteins, vitamins, carnitine, zinc and many others are commonly contaminated with performance enhancing substances (anabolic agents or CNS stimulants). The contaminants in the nutritional supplements include sibutramine, stanozolol, testosterone, caffeine, ephedrine that help in performance enhancement in several ways. So, the doping authorities insist on getting a laboratory test done for checking the doping substances in the nutritional supplements before their usage by the athletes; and also repeating the test for every new batch. Also, they advise on using these products only after consultation with a nutritional expert regarding its beneficial effects and by getting them from companies that are reliable. The detection of these contaminants is done by urine testing. Doping test is considered to be positive if the levels of anabolic steroids in urine are $>10 \mathrm{ppb}$; and for CNS stimulants, it is $>100 \mathrm{ppb}^{23}$

\section{Ergogenic herbs}

The most common herbs used as ergogenic aids are ginseng, ephedrine, caffeine and Eurycoma longifolia Jack. Ginseng possesses anabolic, CNS 
stimulant and antioxidant effects. It thereby helps in the improvement of alertness, increasing the thresholds of stress and fatigue in elite athletes. A minimum dose of $200 \mathrm{mg} /$ day for at least 8 weeks is necessary to exhibit its ergogenic effects. It also helps in enhancing the cardiorespiratory status and increase the exercise duration for exhaustion. Apart from these, it also acts as a cognition enhancer. It is comparatively safe without any teratogenicity or mutagenicity effects. ${ }^{24}$

Ephedrine, a sympathomimetic alkaloid is also found to have ergogenic properties. It increases the strength, reduces fatigue and improves alertness and aerobic performance. The effects of ephedrine are more pronounced when given with caffeine rather than giving it alone. Eurycoma longifolia Jack, another ergogenic herb was found to enhance the muscle strength when administered at a dose of $150 \mathrm{mg}$ for five weeks; and was tried in runners and cyclists as an herbal drink to improve their performance. Also, the safety profile is comparatively wide; it is non-toxic even at a daily dose of about $600 \mathrm{mg}$ in humans. It also has other applications as an anticoagulant, antimalarial, antibacterial, antihyperglycaemic and as an antianxiety drug. ${ }^{24}$

However, herbs as an ergogenic aid are still a debatable concept. Most of these herbs are found to exhibit some positive effects on the psychological states of the individual like mood, stress and mental alertness. Whether these psychological improvements can enhance the performance of athletes needs to be clarified with further studies. ${ }^{24}$

\section{DESIGNER DRUGS IN SPORTS}

The anti-doping agencies have developed efficient methods with a reasonably good sensitivity to help detect the doping substances that are commonly used by the athletes. This has led to the advent of designer drugs that are specifically engineered to evade the doping tests when used in sports and also from being detected when misused for other recreational purposes and pleasure sensations. These drugs are manufactured illegally in the clandestine laboratories with some novel modifications in the already existing drugs at a low cost. Another major concern with the designer drugs is their safety profile which is not known and at times is unpredictable as there are lack of studies confirming their efficacy and safety. The most common drug classes targeted are the anabolic steroids, CNS stimulants, cannabinoids in human sports; and the sildenafil analogs in animal sports. ${ }^{25}$

The anti-doping authorities were first aware of the usage of designer steroids by athletes with the Balco scandal. During this scandal, a new drug Tetrahydrogestrinone (THG), also commonly called as clear; came into limelight. The main intention of designing this is to escape their detection in the doping tests with the currently available methods. It was later found out that THG is not the only compound abused, but also others like the T/ET cream (the cream), erythropoietin, human GH, insulin, modafinil and liothryonine were also given to enhance the performance of the athletes. The program was so planned that they were not detected even in the out-of-competition testing. ${ }^{25}$

The other designer drugs identified include the following:

- $\quad$ Steroids - Norbolethone, desoxymethyltestosterone

Norbolethone was detected after performing detailed laboratory investigations and looking for the alterations in the steroid levels in blood and urine. And desoxymethyltestosterone was found to be misused after the analysis of the seized materials from the athletes.

- SARM's (selective androgen-receptor modulators)- Ostarine (MK2866) andarine (S4)

This class of drugs are structurally different from the AAS, but however share the common benefits of performance enhancement. These drugs are found to have a beneficial role in heart failure and osteoporosis, however due to the serious side-effects associated with these drugs; they were rejected for further pharmaceutical de- velopment. But now they are sold illegally in the black market and are used by athletes to help improve their muscle mass. ${ }^{25}$

- SCRA's (synthetic cannabinoid receptor agonists) - Dronabinol, Nabilone, Nabiximols.

They are generally taken for getting a pleasure sensation, but they also elevate the pain thresholds exhibiting an analgesic action. Many athletes opine it as an ergogenic aid; as it helps reduce anxiety and provide relaxation which can help improve their performance. But the various studies conducted have confirmed that it's only a misconception as it has no additional benefits in sports. Also, some consider it to be an ergolytic; and was found to impair the cognitive functions and psychomotor skills. However, the anti-doping agencies have included these substances in the prohibited list considering their widespread use by the athletes and to avoid the associated health risks. ${ }^{26}$

- Others - ryanodine calastabin complex stabilizers (S-107 and JTV-519), hypoxia-inducible factor [HIF] stabilizer (FG-2216), PPAR (peroxisome proliferator-activated receptor) delta agonists (GW1516).

The usage of designer drugs has widely increased in the recent years due to their availability even on the internet. They are illegally sold by labelling them as "not for human consumption" or as "plant products" so as to avoid the legal issues. And many of these drugs do not actually contain the active ingredient that is claimed in the products. So, for their detection, a variety of newer techniques were being developed in mass spectrometry (MS), immunoassays or with the usage of specific biomarkers. ${ }^{25}$

\section{WORLD ANTI-DOPING AGENCY (WADA)}

The usage of PED's has started to increase at a significant pace in the field of sports. To keep it under check so as to establish a fair competition among the athletes, legislations are needed which should be clear and also universally acceptable. So, with this insight, the IOC (International Olympic Committee) held the first conference to discuss regarding the issues of doping in sports in 1999 and established the WADA. A WADA code was framed, setting a criterion to ban the usage of certain substances if it meets any two of the following criteria-

(i) Enhances performance

(ii) Represents an actual or potential health risk to the athlete

(iii) Its use must be against the "spirit of sport"

WADA is jointly funded by the Olympic organisations and the governments worldwide. Also, the Prohibited List of substances in sports is regularly updated once a year after a thorough discussion with the specialists in various fields considering the pharmaceutical and ethical aspects. It also focuses in the development of newer techniques to expand the detection window of doping substances. The legal actions taken on the athletes when tested positive during the doping tests varies among different nations. In some countries like France, Denmark, Austria, it is even considered to be a criminal offence. ${ }^{27,28}$

\section{WADA PROHIBITED LIST FOR 2019}

WADA releases the prohibited list of doping substances and methods for usage by the athletes every year. The broad classes of substances and the methods prohibited for the year 2019 are shown in Figure $2 .^{29}$

The information regarding the usage of new substances and methods by the athletes is an obvious concern for their inclusion in the prohibited list. This information is obtained by collaboration with the pharmaceutical companies, gathering information and evidence if any from the customs officers and police, from the journalists, whistle-blowers and also from the athletes when found guilty. WADA strictly regulates the usage of these substances, not alone to have a fair competition in sports; but 
Table 1: Drug classes of commonly used PED's. Anabolic agents.

Anabolic androgenic steroids (AAS) - testosterone, nandrolone, stanozolol androstenedione, trenbolone, boldenone

Others - Clenbuterol

Hormonal substances - Erythropoietin, growth hormone, insulin, human chorionic gonadotropin (hCG), luteinizing hormone $(\mathrm{LH})$

CNS stimulants - Caffeine, ephedrine, amphetamine, methylphenidate, nikethamide, amiphenazole, strychnine, cocaine

$\boldsymbol{\beta}_{2}$ agonists - Salbutamol, salmeterol, formeterol

$\boldsymbol{\beta}$ blockers - Propranolol

Diuretics and masking agents- Hydrochlorthiazide, frusemide, desmopressin, probenecid, amiloride, metolazone

Narcotics - Codeine, morphine, fentanyl, buprenorphine

Blood doping (Blood boosters)

Gene doping

Nutritional supplements - Creatine, $\beta$-hydroxyl $\beta$-methyl butyric acid

Ergogenic herbs - Ginseng, caffeine, Eurycoma longifolia Jack

Miscellaneous - Cyclooxygenase-2 (COX-2) inhibitors, tamoxifen,

bromantane

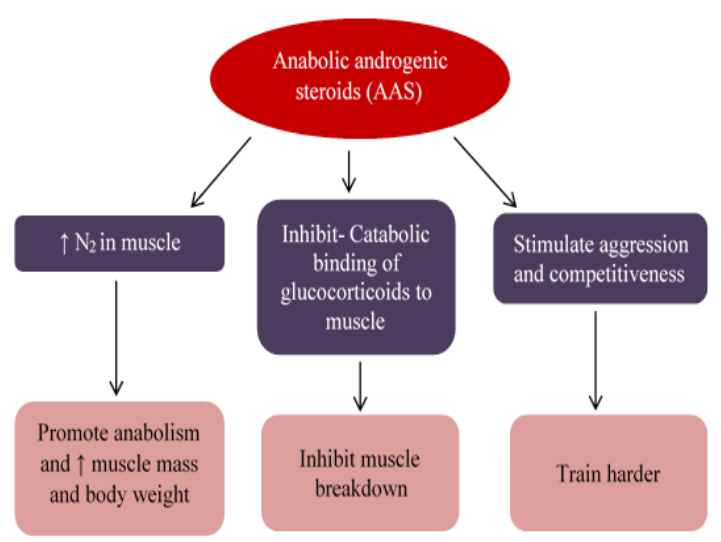

Figure 1: Mechanism of action of anabolic androgenic steroids (AAS).

also to prevent them from the associated adverse effects as many of these are easily available in the black markets and there are no proper clinical studies to ensure their safety. ${ }^{28}$

\section{ATHLETE BIOLOGICAL PASSPORT (ABP)}

The traditional analyses of doping substances in the biological fluids of athletes is not always feasible and do has some limitations. The usage of erythropoiesis stimulating agents, autologous blood transfusion, designer drugs and even the manipulation of urine has made the detection process quite troublesome in many instances. So, the anti-doping agencies have made a cut-off of the upper limits of haemoglobin and haematocrit levels during dope testing; but there are various concerns associated with the inclusion of these parameters like the influence of age, gender, ethnicity and also external factors (exercise, high altitudes and body posture). The authorities then recommended for the collection of longitudinal blood profiles considering the external factors to develop an adaptive model based on the individual's blood profile that are later reviewed by experts. ${ }^{20}$

The passport data of the athletes is interpreted on a regular basis so as to identify the specific targets which can in turn help in the refinement of available analytical methods for the strengthening of anti-doping measures. The data storage, reporting and sharing is done by WADA's ADAMS (Anti-Doping Administration and Management System). AD-

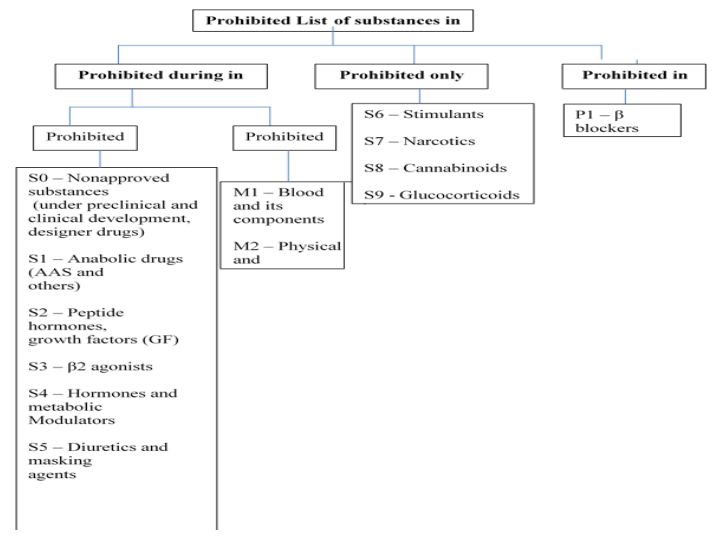

Figure 2: WADA Prohibited List of substances and methods for 2019.

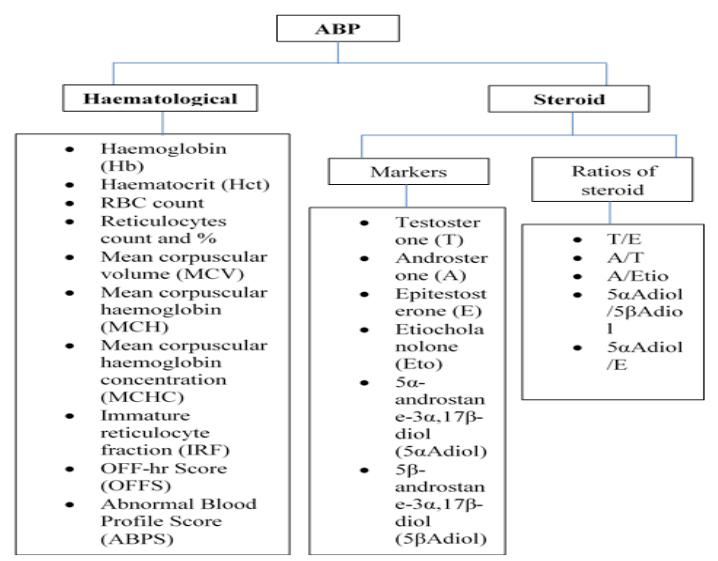

Figure 3: Modules of ABP.

AMS consists of an APMU (Athlete Passport Management Unit) that gives a review till date of an athlete's passport. $\mathrm{ABP}$ contains two modules to analyse the doping substances which are analysed in the WADA accredited laboratory - the haematological module and the steroid module which are shown in detail in Figure $3 .^{30}$

\section{CONCLUSION}

The use of PED's like the anabolic steroids, CNS stimulants and many other substances among the athletes has drastically increased and is expected to rise further in the coming years due to the advancements in science and technology. This has become a great challenge for antidoping agencies to prevent the misuse of certain drugs which could lead to unfair advantage amongst the athletes. WADA was established to cope up with these issues and it has framed a set of universally acceptable, clear and transparent guidelines updating them periodically. But, down the line emergence of designer drugs has become another hurdle to overcome. Moving forward, $\mathrm{ABP}$ was implemented to resolve these issues and also to prevent the athletes from the unforeseen adverse effects of such substances. Though several measures are being taken constantly to keep it under check, the newer developments may result in newer drugs that could be misused. So, it's the responsibility of the athletes and also the physicians to behave morally; and legislations should be put forward in a stringent manner to avoid such acts in the future.

\section{CONFLICT OF INTEREST}

None to declare. 


\section{ABBREVIATIONS}

ABP: Athlete Biological Passport; ADAMS: Anti-Doping Administration and Management System; APMU: Athlete Passport Management Unit; PED’s: Performance Enhancing Drugs; WADA: World Anti-Doping Agency.

\section{REFERENCES}

1. Effects of performance enhancing drugs. U.S. Anti-Doping Agency. 2018. Available at https://www.usada.org/substances/effects-of-performance-enhancingdrugs/

2. Frati P, Kyriakou C, Del RA, Marinelli E, Vergallo GM, Zaami S. Smart drugs and synthetic androgens for cognitive and physical enhancement: Revolving doors of cosmetic neurology. Curr Neuropharmacol. 2015;13(1):5-11.

3. Rang HP, Ritter JM, Flower RJ, Henderson G. Lifestyle drugs and drugs in sport. In: Rang and Dale's pharmacology Eighth edition. UK, Churchill Livingstone. 2016;703-6

4. Dandoy C, Gereige RS. Performance-Enhancing Drugs. Pediatr Rev. 2012;33(6):265-72

5. Harris W. 10 performance-enhancing drugs that aren't steroids. 2018. Available at https://science.howstuffworks.com/10-performance-enhancing-drugs4.htm

6. Chen CK, Muhamad AS, Ooi FK. Herbs in exercise and sports. J Physiol Anthropol. 2012;31(1):4.

7. Spann C, Winter ME. Effect of clenbuterol on athletic performance. Ann Pharmacother. 1995;29(1):75-7.

8. Peng SH, Segura J, Farre M, Gonzalez JC, Torre DLX. Plasma and urinary markers of oral testosterone undecanoate misuse. Steroids. 2002;67(1):39-50.

9. Basaria S. Androgen abuse in athletes: Detection and consequences. J Clin Endocrinol Metabol. 2010;95(4):1533-43.

10. Andrews MA, Magee CD, Combest TM, Allard RJ, Douglas KM. Physical effects of anabolic-androgenic steroids in healthy exercising adults: A systematic review and meta-analysis. Curr Sports Med Rep. 2018;17(7):232-41.

11. Rang HP, Ritter JM, Flower RJ, Henderson G. Haemopoietic system and treatment of anaemia. In: Zeigler M, Mortimer A. Rang and Dale's pharmacology Eighth edition. UK, Churchill Livingstone. 2016;308-16.

12. Robinson N, Giraud S, Saudan C, Baume N, Avois L, Mangin P. Erythropoietin and blood doping. Br J Sports Med. 2006;40(suppl 1):i30-4

13. Baumann GP. Growth hormone doping in sports: A critical review of use and detection strategies. Endocr Rev. 2012;33(2):155-86.
14. Holt RIG, Sonksen PH. Growth hormone, IGF-I and insulin and their abuse in sport. Br J Pharmacol. 2008;154(3):542-56.

15. Kuuranne T, Ahola L, Pussinen C, Leinonen A. Analysis of human chorionic gonadotropin (hCG): application of routine immunological methods for initial testing and confirmation analysis in doping control. Drug Test Anal. 2013;5(8):614-8

16. Thevis M, Sigmund G, Geyer H, Schanzer W. Stimulants and doping in sport. Endocrinol Metab Clin North Am. 2010;39(1):89-105.

17. Kindermann $W$, Meyer T. Inhaled $\beta_{2}$ agonists and performance in competitive athletes. Br J Sports Med. 2006;40(suppl 1):i43-7.

18. Cadwallader A, DeLa TX, Tieri A, Botre F. The abuse of diuretics as performanceenhancing drugs and masking agents in sport doping: Pharmacology, toxicology and analysis. Br J Pharmacol. 2010;161(1):1-16.

19. Seif-Barghi T, Moghadam N, Kobarfard F. Morphine/Codeine ratio, a key in investigating a case of doping. Asian J Sports Med. 2015;6(4):e28798.

20. Jelkmann W, Lundby C. Blood doping and its detection. Blood. 2011;118(9):2395 404

21. Spahn DR. Blood substitutes, artificial oxygen carriers: Perfluorocarbon emulsions. Crit Care. 1999;3(5):R93-7.

22. Neuberger EWI, Simon P. Gene and cell doping: The new frontier - beyond myth or reality. Med Sport Sci. 2017;62:91-106.

23. DeHon O, Coumans B. The continuing story of nutritional supplements and doping infractions. Br J Sports Med. 2007;41(11):800-5.

24. Chen C, Muhamad A, Ooi F. Herbs in exercise and sports. J Physiol Anthropol. 2012;31(1):4.

25. Teale P, Scarth J, Hudson S. Impact of the emergence of designer drugs upon sports doping testing. Bioanalysis. 2012;4(1):71-88

26. Ware M, Jensen D, Barrette A, Vernec A, Derman W. Cannabis and the health and performance of the elite athlete. Clin J Sport Med. 2018;28(5):480-4

27. Negro M, Marzullo N, Caso F, Calanni L, D'Antona G. Opinion paper: Scientific philosophical and legal consideration of doping in sports. Eur J Appl Physiol. 2018;118(4):729-36.

28. Athanasiadou I, Voss S, Lyris E, Aljaber A, Alsayrafi M, Georgakopoulos C. An alytical progresses of the World Anti-Doping Agency Olympic laboratories: a 2016 update from London to Rio. Bioanalysis. 2016;8(21):2265-79.

29. The prohibited list January 2019. World Anti-Doping Agency. 2018. Available at https://www.wada-ama.org/sites/default/files/wada_2019_english_prohibited_list.pdf

30. Athlete biological passport operating guidelines. World Anti-Doping Agency 2018;61. https://www.wada-ama.org/sites/default/files/resources/files/guidelines_abp_v61_2018_jul_en.pdf

Article History: Submission Date : 17-09-2019; Revised Date : 15-10-2019; Acceptance Date : 22-10-2019

Cite this article: Sowjanya K, Girish C. An Overview of Performance Enhancing Drugs (PED's) in Sports and WADA. J Young Pharm. 2019;11(4):344-9. 